BULLETIN OF THE

AMERICAN MATHEMATICAL SOCIETY

Volume 78, Number 4, July 1972

\title{
AZUMAYA, SEMISIMPLE AND IDEAL ALGEBRAS 1
}

\author{
BY M. L. RANGA RAO \\ Communicated by Dock S. Rim, January 31, 1972
}

Introduction. If $\boldsymbol{A}$ is an algebra over a commutative ring, then for any ideal $I$ of $R$ the image of the canonical map $I \otimes_{R} A \rightarrow A$ is an ideal of $A$, denoted by $I A$ and called a scalar ideal. The algebra $A$ is called an ideal algebra provided that $I \rightarrow I A$ defines a bijection from the ideals of $R$ to the ideals of $A$. The contraction map defined by $\mathfrak{A} \rightarrow \mathfrak{A} \cap R$, being the inverse mapping, each ideal of $A$ is a scalar ideal and every ideal of $R$ is a contraction of an ideal of $A$. It is known that any Azumaya algebra is an ideal algebra (cf. [2], [4]).

In this paper we initiate the study of ideal algebras and obtain a new characterization of Azumaya algebras. We call an algebra finitely generated (or projective) if the $R$-module $A$ is, and show that a finitely generated algebra $A$ is Azumaya iff its enveloping algebra is ideal (1.7).

Hattori introduced in [9] the notion of semisimple algebras over a commutative ring as those algebras whose relative global dimension $[\mathbf{1 0}]$ is zero. If $R$ is a Noetherian ring, central, finitely generated semisimple algebras have the property that their maximal ideals are all scalar ideals [6, Theorem 1.6]. It is also known that for $R$, a Noetherian integrally closed domain, finitely generated, projective, central semisimple algebras are maximal orders in central simple algebras [9, Theorem 4.6]. Therefore by Remark 2.4, over Dedekind domains finitely generated, central, projective semisimple algebras are ideal algebras. (The converse is trivial by $[5,8.1]$.) The Endo-Watanabe example of a semisimple algebra over a discrete valuation ring that is not Azumaya [7, Theorem 1] then assures that the class of finitely generated, central ideal algebras is strictly larger than that of Azumaya algebras. Over artinian rings, however, finitely generated central ideal algebras are Azumaya (Corollary 1.10).

Semisimple algebras need not be projective over their centers even if the centers are artinian [7] and, hence, not all semisimple algebras are ideal algebras. However, as mentioned above, over artinian rings or over Dedekind domains, finitely generated, central projective semisimple algebras are precisely ideal algebras.

We do not know whether or not, over a Noetherian ring, every finitely

AMS 1970 subject classifications. Primary 16A16; Secondary 16A18, 16A60.

Key words and phrases. Noetherian rings, integrally closed domains, Dedekind domains, scalar ideals, Azumaya algebras, ideal algebras, separable algebras, discrete valuation ring, relative homological dimension, faithfully flat modules, semisimple algebras, maximal orders.

1 These results are part of the author's doctoral dissertation written under the direction of Professor Carl Faith at Rutgers, The State University, New Brunswick, New Jersey. 
generated, projective, central semisimple algebra is an ideal algebra; but although an easy computation shows that in this case, every prime ideal of the algebra is a scalar ideal. Nevertheless, it appears unlikely that this should imply in the general case that every ideal is scalar.

For $R$ a Noetherian ring, we obtain a homological property for a finitely generated ideal algebra $A$, namely $\mathrm{gl} \operatorname{dim} A=\operatorname{gl} \operatorname{dim} R$ (Proposition 2.2). If we further restrict $R$ to be a Noetherian integrally closed domain with the quotient field $K$, then any finitely generated central ideal algebra $A$ is a maximal order in the central simple algebra $K \otimes_{R} A$ (Proposition 2.3). It is this fact above all others which arouses interest in ideal algebras in as much as they are undoubtedly a new class of maximal orders.

It is known that, over a Noetherian integrally closed domain, a projective finitely generated central algebra $A$ is separable iff for all prime ideals $p$ of height $1, A_{p}$ is $R_{p}$ separable $[2,4.6]$. This property does not hold in ideal algebras $[7, \S 3]$.

In order to publish the main ideas and results as rapidly as possible we have suppressed the details which may be found in the author's thesis.

The author expresses his gratitude to Dr. Carl Faith for the direction and preparation of this paper.

1. Ideal algebras and Azumaya algebras. Jensen [11] has shown that if $R$ (not necessarily commutative) is a ring without proper zero divisors, a torsion free left $R$-module $M$ is flat iff $I M \cap J M=(I \cap J) M$ for all right ideals $I$ and $J$ of $R$. The same type of proof in fact yields

LEMMA 1.1. For an arbitrary ring $R$, and a left $R$-module $M$ the following are equivalent:

(a) $M$ is $R$-flat.

(b) (1) $I M \cap J M=(I \cap J) M$ for all right ideals $I$ and $J$ of $R$.

(2) $(0: a)_{R} M=(0: a)_{M}$, where $(0: a)_{R}=\{r \in R \mid a r=0\}$ and $(0: a)_{M}$ $=\{m \in M \mid a m=0\}$

Proposition 1.2. If $A$ is an ideal algebra over $R$, then $A$ is faithfully flat over $R$.

Proof. From the definition of ideal algebras it is clear that the conditions (b) of the lemma are satisfied and hence $A$ is $R$-flat. As $m A \neq A$ for all maximal ideals $m$ of $R, A$ is faithfully flat over $R$.

COROLLARY 1.3. If $R$ is either Noetherian or a domain, a finitely generated ideal algebra $A$ is faithfully projective over $R$.

We now give two criteria for separability.

PROPOSITION 1.4. If $R$ is a ring such that $R / m$ is perfect for all maximal ideals $m$ of $R$ then a finitely generated ideal algebra is separable. 
Proof. $A / m A$ is simple over $R / m$ and therefore is separable by the perfection of $R / m$. Our proposition now follows from Endo-Watanabe $[8,1.1]$.

It is easy to verify the next lemma.

LEMMA 1.5. An algebra $A$ is an ideal algebra iff $A_{m}$ is an ideal $R_{m}$-algebra for all maximal ideals $m$ of $R$.

LEMMA 1.6. Let $(R, m)$ be a local ring and $B \subset A$ be two finitely generated ideal algebras such that $\operatorname{Dim}_{R} B=\operatorname{Dim}_{R} A$. Then $B=A$.

Proof. Obviously $m A \cap B \supset m B$. The unique maximal ideal of $B$ is $m B$, hence $m A \cap B=m B$. The natural map $\eta: B / m B \rightarrow A / m A$ induced by the inclusion $B \subset A$ is an injection. Since $\operatorname{Dim}_{R} B=\operatorname{Dim}_{R} A, \eta$ is a surjection. By Nakayama's lemma, $B=A$.

Proposition 1.7. A finitely generated algebra $A$ is Azumaya iff the enveloping algebra $A^{e}$ is an ideal algebra.

Proof. If $A$ is Azumaya so is $A^{e}$, and hence $A^{e}$ is an ideal algebra. Conversely because of Lemma 1.5 , it is sufficient to assume that $(R, m)$ is a local ring. Since $A$ is an $R$-direct summand of $A^{e}$, then $A$ is $R$-free (Proposition 1.2). Hence $\operatorname{End}_{R} A$ is Azumaya, and, in particular, an ideal algebra. Consider the canonical algebra map $\eta: A^{e} \rightarrow \operatorname{End}_{R} A$. This is an injection since every ideal of $A^{e}$ is a scalar ideal. Moreover, $\operatorname{Dim}_{R} A^{e}$ $=\operatorname{Dim}_{R}$ End $A=n^{2}$, where $n$ is the dimension of $A$ as an $R$-module. Thus, by Lemma 1.6, $\eta$ is an isomorphism, and, therefore, $A$ is Azumaya [4, Chapter 3, 4.1].

Along the same lines one can show

Proposition 1.8. If $A$ is a finitely generated ideal algebra over $R$, then every algebra endomorphism of $A$ is an automorphism.

Proposition 1.9. If $R$ is a commutative artinian local ring and $A$ is a central finitely generated and projective algebra with a unique maximal ideal $m A$, then $A$ is Azumaya.

See [6, Proposition 2.1].

COROLLARY 1.10. For a commutative artinian ring $R$, a finitely generated central ideal algebra is Azumaya.

Proof. Follows from Lemma 1.5 and Corollary 1.3.

Since there exist ideal algebras that are not Azumaya (Introduction), then by 1.7 , the tensor product of two ideal algebras need not be an ideal algebra. However, one can prove

Proposition 1.11. If $A$ and $B$ are algebras such that one of them is flat over $R$ and $A \otimes_{R} B$ is an ideal algebra then $A$ and $B$ are ideal algebras. 
Proposition 1.12. If $S$ is a commutative $R$-algebra such that $S$ is $R$ faithfully flat and $S \otimes_{R} A$ is an $S$-ideal algebra then $A$ is an $R$-ideal algebra.

2. Ideal algebras and maximal orders. The following proposition is due to Silver [12].

Proposition 2.1. Let $(R, m)$ be a Noetherian local ring and $A$ be a finitely generated projective algebra with the Jacobson radical $m A$. Then $\mathrm{gl} \operatorname{dim} A=\mathrm{gl} \operatorname{dim} \boldsymbol{R}$.

Proof. Follows immediately from $[12,4.6$ and 4.9].

Proposition 2.2. For $R$ a Noetherian ring and $A$ a finitely generated ideal algebra, $\mathrm{gl} \operatorname{dim} A=\mathrm{gl} \operatorname{dim} R$.

Proof. Since

$$
\operatorname{gl} \operatorname{dim} A=\operatorname{Sup}_{m}\{\mathrm{gl} \operatorname{dim} A \mid m, \text { maximal ideals of } R\},
$$

it is sufficient to assume $(R, m)$ to be a local ring. The proposition now follows from 1.3 and 2.1 .

If $R$ is a domain and $A$ is a finitely generated central ideal algebra, then $A$ can be imbedded in $K \otimes A$, a central simple algebra. As an immediate consequence of the above proposition and [1, Theorem 1.5 and Theorem 2.3] we have the following:

Proposition 2.3. Over a Noetherian integrally closed domain, a finitely generated central ideal algebra is a maximal order in a central simple algebra.

RemarK 2.4. It is known that for $R$ a Dedekind domain, every ideal of a maximal order in a central simple algebra is a product of maximal ideals [3, Theorem 8.6]. Hence if the maximal ideals are scalar ideals, then every ideal is scalar. In fact, in this case, $R$ will be an ideal algebra since every ideal of $R$ is a contraction of an ideal of the algebra because of faithful projectivity.

RemarK 2.5 . Let $(R, m)$ be a discrete valuation ring and $A$ be a finitely generated central ideal algebra. $A$ being a maximal order, its $m$-adic completion $\hat{A}$ is also a maximal $\hat{R}$-order in a central simple algebra with unique maximal scalar ideal. Hence by the above remark, $\hat{A}$ is also a central $\hat{R}$-ideal algebra. As the separability of $\hat{A}$ implies the separability of $A$, we remark that even over complete discrete valuation rings, finitely generated central ideal algebras need not be Azumaya. (See the Introduction.)

\section{REFERENCES}

1. M. Auslander and O. Goldman, Maximal orders, Trans. Amer. Math. Soc. 97 (1960), 1-24. MR $22 \# 8034$. 
2. The Brauer group of a commutative ring, Trans. Amer. Math. Soc. 97 (1960), 367-409. MR 22 \# 12130.

3. H. Bass, Algebraic K-theory, Benjamin, New York, 1968. MR 40 \# 2736.

4. - Lectures on topics in algebraic $K$-theory, Tata Notes, no. 41 , Bombay.

5. S. Endo, Completely faithful modules and quasi-Frobenius algebras, J. Math. Soc. Japan 19 (1967), 437-456. MR 37 \# 1406.

6. S. Endo and Y. Watanabe, The centers of semisimple algehras over a commutative ring, Nagoya Math. J. 30 (1967), 285-293. MR 35 \# 4256.

7. - The centers of semisimple algebras over a commutative ring. II, Nagoya Math. J. 39 (1970), 1-6. MR 41 \# 8461.

8. - On separable algebras over a commutative ring, Osaka J. Math. 4 (1967), 233-242. MR 37 \#2796.

9. A. Hattori, Semisimple algebras over a commutative ring, J. Math. Soc. Japan 15 (1963), 404-419. MR 28 \# 2125.

10. G. Hochschild, Relative homological algebra, Trans. Amer. Math. Soc. 82 (1956), 246-269. MR 18, 278.

11. Chr. U. Jensen, $A$ remark on flat and projective modules, Canad. J. Math. 18 (1966), 943-949. MR 33 \# 7375.

12. L. Silver, Noncommutative localizations and applications, J. Algebra 7 (1967), 44-76. MR 36 \# 205.

Department of Mathematics, Rutgers, the State University, New Brunswick, NEW JERSEY 08903

Current address: Department of Mathematics, University of Virginia, Charlottesville, Virginia 22903 